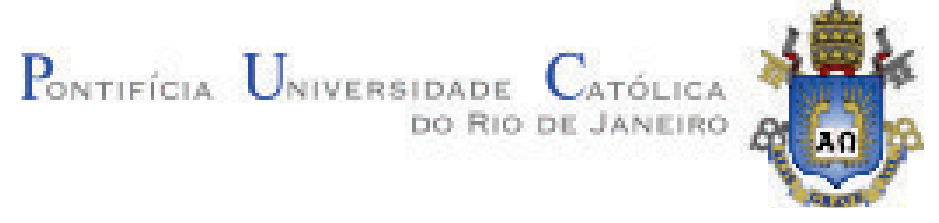

Patricia Emidio de Azevedo

Deslocamento de líquidos não newtonianos em células de Hele-Shaw

Dissertação de Mestrado

Dissertação apresentada como requisito parcial para obtenção do grau de Mestre pelo Programa de Pós-graduação em Engenharia Mecânica do Departamento de Engenharia Mecânica da PUCRio

Orientador: Prof. Paulo Roberto de Souza Mendes 
Patricia Emidio de Azevedo

\section{Deslocamento de líquidos não newtonianos em células de Hele-Shaw}

Dissertação apresentada como requisito parcial para obtenção do grau de Mestre pelo Programa de Pós-graduação em Engenharia Mecânica do Departamento de Engenharia Mecânica da PUCRio. Aprovada pela Comissão Examinadora abaixo assinada.

Prof. Paulo Roberto de Souza Mendes Orientador Departamento de Engenharia Mecânica - PUC-Rio

Prof. Paulo Roberto de Souza Mendes Departamento de Engenharia Mecânica - PUC-Rio

Prof. Márcio da Silveira Carvalho Departamento de Engenharia Mecânica - PUC-Rio

Prof. Mônica Feijó Naccache Departamento de Engenharia Mecânica - PUC-Rio

Prof. José Eugênio Leal Coordenador Setorial do Centro Técnico Científico - PUC-Rio 
Todos os direitos reservados. É proibida a reprodução total ou parcial do trabalho sem autorização da universidade, do autor e do orientador.

\section{Patricia Emidio de Azevedo}

Graduou-se em Engenharia Mecânica na Pontifícia Universidade Católica do Rio de Janeiro em 2011.

Ficha Catalográfica

Azevedo, Patricia Emidio de

Deslocamento de líquidos não newtonianos em células de Hele-Shaw / Patricia Emidio de Azevedo; orientador: Paulo Roberto de Souza Mendes. - 2015.

136 f.: il. (color.); $30 \mathrm{~cm}$

Dissertação ( mestrado) - Pontifícia Universidade Católica do Rio de Janeiro, Departamento de Engenharia Mecânica, 2015.

Inclui bibliografia

1. Engenharia mecânica - Teses. 2. Células de Hele-Shaw. 3. Invasão de fluido de perfuração. 4. Estabilidade de interface.

5. Fluido não newtoniano. I. Mendes, Paulo Roberto de Souza.

II. Pontifícia Universidade Católica do Rio de Janeiro. Departamento de Engenharia Mecânica. III. Título. 


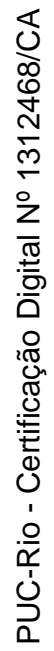

Aos meus pais e meu noivo 


\section{Agradecimentos}

Gostaria primeiramente de agradecer ao meu orientador, professor Paulo Roberto de Souza Mendes, pela oportunidade e confiança depositada em mim para realizarmos em conjunto essa pesquisa.

A minha família pelo apoio e carinho. Agradeço, em especial, à minha mãe e ao meu pai que sempre me apoiaram, me incentivaram e me ajudaram de todas as maneiras possíveis para a realização desse sonho, sofrendo em cada decepção e vibrando por cada sucesso.

Ao meu noivo, melhor amigo e companheiro de todas as horas, Marcelo Silval, pelo carinho, compreensão, amor e solidariedade. Obrigada por estar ao meu lado durante esses nove anos, como amigo, namorado e depois como noivo, sempre me apoiando e me ajudando.

Obrigada a todos do Grupo de Reologia da PUC-Rio e amigos. Ao Bruno Fonseca, um irmão, que me ajudou nos momentos mais difíceis tanto emocionalmente quanto profissionalmente. Ao Alexandre Fernandes com sua experiência de vida que sempre me motivou e encorajou. Agradeço a eles também por me auxiliarem na montagem e manutenção da bancanda experimental. À Caroline Grossi, técnica em química do laboratório e amiga, que me ajudou no preparo dos fluidos e na caracterização reológica dos mesmos, além de ajudar a solucionar problemas os quais não dominava devido a minha formação acadêmica. À Paula Nascimento, estagiária do laboratório e amiga, que com sua proatividade sempre me ajudou a solucionar problemas decorrentes e me ouvia sempre que necessário. À Deborah Sanches, uma amiga que me apoiou e me ajudou nos momentos mais difíceis, sempre me auxiliando a tomar as decisões corretas.

Agradeço a todos os meus colegas do mestrado da PUC-Rio, em destaque: Behbood Abedi, William Lopez e Himer Mieles. Obrigada pelas boas risadas e descontração na sala da pós-graduação, além da ajuda nas matérias do curso.

A todos os professores que trabalharam para que eu pudesse ter uma formação de excelêcia, em destaque: professor Luis Fernando Azevedo; professor Márcio Carvalho; professora Mônica Naccache; professor Paulo Roberto de Souza Mendes; professor Ivan Fabio Mota de Menezes; professor João Carlos Ribeiro Plácido e professor Geraldo Spinelli Ribeiro.

A todos do Departamento de Engenharia Mêcanica pela colaboração no decorrer desses dois anos.

À Petrobras, PUC-Rio e ao CAPES, pelo auxílio concedido, sem os quais esse trabalho não poderia ter sido realizado. 


\section{Resumo}

Azevedo, Patricia Emidio de; Mendes, Paulo Roberto de Souza. Deslocamento de líquidos não newtonianos em células de Hele-Shaw. Rio de Janeiro, 2015. 136p. Dissertação de Mestrado - Departamento de Engenharia Mecânica, Pontifícia Universidade Católica do Rio de Janeiro.

A perfuração de poços de petróleo, principalmente em ambientes offshore, é uma operação que demanda um custo muito elevado, portanto a minimização de problemas e danos ao reservatório produtor é muito importante. $\mathrm{O}$ projeto de um fluido de perfuração que garanta a mínima invasão na rocha reservatório é um tópico fundamental, como consequência a indústria petrolífera tem investido em estudos com o intuito de evitar que este problema ocorra. Sendo assim, nesse trabalho estudou-se o escoamento de fluidos em um meio poroso ideal, a célula de Hele-Shaw. Para realizar esse trabalho foram utilizados dois fluidos não newtonianos: uma solução aquosa de um polímero flexível, a poliacrilamida; e uma solução aquosa de um polímero rígido, a goma xantana. Esses fluidos não newtonianos representaram o fluido de perfuração, já o papel do petróleo presente na rocha reservatório foi desempenhado pelo óleo mineral. A partir desse experimento é possível observar a instabilidade de Saffman-Taylor ou viscous fingers, que é um fenômeno observado quando um fluido de menor viscosidade desloca outro de maior viscosidade. Esse fenômeno é muito importante em diversas aplicações, tais como: invasão de fluido de perfuração em meios porosos (caso que será estudado), recuperação secundária e terciária de petróleo, fraturamento hidráulico, processamento de polímeros, hidrologia e filtração. $\mathrm{O}$ experimento consiste em analisar a evolução da interface entre os dois fluidos através de uma câmera filmadora. A partir das imagens é determinada qualitativamente a eficiência do deslocamento. Com o conjunto de imagens é possível observar também em quais parâmetros dinâmicos e reológicos ocorre a transição fingers (interface instável) para plug (interface estável), e viceversa. Também foram realizados testes reológicos nos quais foi possível conhecer o comportamento dos fluidos não newtonianos utilizados. Com base nos dados coletados observou-se que tanto para a poliacrilamida quanto para a goma xantana a transição ocorre a uma taxa de cisalhamento adimensional próxima de 3.

\section{Palavras-chave}

Célula de Hele-Shaw; Invasão de fluido de perfuração; Estabilidade de interface; Fluido não newtoniano; 


\section{Abstract}

Azevedo, Patricia Emidio de; Mendes, Paulo Roberto de Souza (Advisor). Liquid-liquid displacement flows in a Hele-Shaw cell including non-Newtonian effects. Rio de Janeiro, 2015. 136p. MSc Dissertation Departamento de Engenharia Mecânica, Pontifícia Universidade Católica do Rio de Janeiro.

The drilling of oil wells, particularly in offshore environments, is an operation which demands a high cost, thus, the minimization of problems and damage to the producing reservoir is very important. The design of a drilling fluid that ensures minimum invasion into the reservoir rock is a major topic, consequently the oil industry has invested in research in order to prevent this problem from occurring. Therefore, this research studied the displacement of fluids in an ideal porous medium, a Hele-Shaw cell. To accomplish this task, the following two non-Newtonian fluids were used: an aqueous solution of a flexible polymer, polyacrylamide; and the other an aqueous solution of a rigid polymer, xanthan gum. Such non-Newtonian fluids represent the drilling fluid, while the function of oil present in the reservoir rock was represented by mineral oil. From this experiment it is possible to observe the Saffman-Taylor instability or viscous fingers, which is a phenomenon observed when a low viscosity fluid displaces a higher viscosity fluid. This phenomenon is very important in many applications, such as drilling fluid invasion in porous media, secondary and tertiary oil recovery, hydraulic fracturing, polymer processing, hydrology and filtration. The experiment consists in analyzing the evolution of the interface between two fluids through a digital camera, and determining the displacement efficiency qualitatively from the imagens. With the set of images is possible to observe in which dynamic and rheological parameters the transition from fingers (unstable interface) to plug (stable interface) occurs, and vice versa. Also, rheological tests were performed in which it was possible to know the behavior of non-Newtonian fluids used in the study. From the data collected it was observed that both the polyacrylamide and for xanthan gum transition occurs near the dimensionless shear rate of 3 .

\section{Keywords}

Hele-Shaw cell; Invasion and drilling fluid; Viscous fingering; nonNewtonian fluid; 


\section{Sumário}

1 Introdução $\quad 21$

1.1 Motivação 21

1.2 Objetivos 24

$\begin{array}{lll}1.3 & \text { Revisão bibliográfica } & 24\end{array}$

2 Metodologia Experimental $\quad 34$

2.1 Fluidos 34

2.2 Bancada experimental 34

2.3 Procedimento experimental 42

3 Formulação matemática $\quad 48$

3.1 Equacionamento 48

3.2 Análise Adimensional 54

3.3 Analogia entre uma célula de Hele-Shaw homogênea e um meio poroso homogêneo 57

ङ 4 Resultados $\quad 59$

4.1 Caracterização reológica 59

4.2 Experimentos de visualização $\quad 74$

5 Conclusões $\quad 122$

5.1 Conclusões 122

$\begin{array}{ll}5.2 \text { Recomendações para futuras pesquisas } & 123\end{array}$

$\begin{array}{lr}\text { Referências } & 124\end{array}$

$\begin{array}{lr}\text { A Goma Xantana } & 129\end{array}$

$\begin{array}{ll}\text { B Poliacrilamida } & 131\end{array}$

$\begin{array}{lrl}\text { C Reologia } & 132\end{array}$ 


\section{Lista de figuras}

1.1 Esquema da invasão do fluido de perfuração na rocha reservatório. 22

1.2 Esquema da invasão do fluido de perfuração na rocha reservatório. 22

1.3 Esquema da célula de Hele-Shaw (38). 25

1.4 Representação da instabilidade de Saffman-Taylor ou viscous fingering. 26

1.5 Testes Homsy. 28

2.1 Primeira versão da célula de Hele-Shaw. 35

2.2 Segunda versão da célula de Hele-Shaw. 35

2.3 Terceira versão da célula de Hele-Shaw.. 36

2.4 Quarta versão da célula de Hele-Shaw.. 36

2.5 Quinta versão da célula de Hele-Shaw. 36

2.6 Sexta versão da célula de Hele-Shaw. 37

2.7 Sétima versão da célula de Hele-Shaw. 37

2.8 Oitava versão da célula de Hele-Shaw. 37

2.9 Dimensões da célula de Hele-Shaw. 39

2.10 Imagem explodida da Célula de Hele-Shaw. 39

2.11 Detalhes da célula de Hele-Shaw. 40

2.12 Célula de Hele-Shaw. 40

2.13 Bancada experimental. 41

2.14 Posição favorável ao escoamento. $\quad 42$

2.15 Posição desfavorável ao escoamento. 42

2.16 Bomba peristáltica da Cole-parmer. 42

2.17 Controlador da bomba peristáltica da Cole-parmer. 43

2.18 Interface do programa Fiji. 44

2.19 (a) Imagem original (b) Área de corte (c) Imagem após seção cortada. 44

2.20 (a) Imagem original (b) Imagem aplicada o filtro passa baixa. 45

2.21 Etapas para binarização das imagens. 45

2.22 Última imagem analisada, $\epsilon=54,56 \%$ do óleo deslocando a poliacrilamida $0,25 \%$. 46

2.23 Óleo deslocando PHPA 0,25\% (a) Imagens antes de serem binarizadas. (b) Imagens binarizadas. $\quad 46$

2.24 Esquema do procedimento experimental. $\quad 47$

3.1 Desenho esquemático da célula de Hele-Shaw. 48

3.2 Modelo adimensional - Fluido newtoniano deslocando fluido não newtoniano. 56

3.3 Modelo adimensional - Fluido não newtoniano deslocando fluido newtoniano. 56

3.4 Modelo da seção de Hele-Shaw. 57

4.1 (a) Reômetro DHR (Discovery Hybrid Rheometers) da TA Instruments (b) Peltier de placas e geometria cônica de $60 \mathrm{~mm} \mathrm{e} 1^{\circ}$ (c)

Peltier de cilindros concêntricos e geometria de cilindros concêntricos. 59

4.2 Curva de escoamento (Flow curve) do óleo mineral - T $=25{ }^{\circ} \mathrm{C} . \quad 61$ 
4.3 Rampa de temperatura (Temperature ramp) do óleo mineral.

4.4 Varredura da amplitude de tensão (Stress sweep) do óleo mineral $\mathrm{T}=25^{\circ} \mathrm{C}$.

4.5 Varredura da amplitude de tensão (Stress sweep) da poliacrilamida $0,25 \%$.

4.6 Varredura da amplitude de tensão (Stress sweep) da goma xantana $0,4 \%$.

4.7 Varredura de tempo (Time sweep) da poliacrilamida 0,25\%.

4.8 Varredura de tempo (Time sweep) da goma xantana $0,40 \%$.

4.9 Varredura de frequência (Frequency sweep) da poliacrilamida 0,25\%. 66

4.10 Varredura de frequência(Frequency sweep) da goma xantana 0,4\%. 66

$4.11 \mathrm{O}$ comportamento de fluido não newtoniano viscoelástico generalizado variando a frequência.

4.12 Taxa de cisalhamento constante $-\dot{\gamma}=0,011 / s$ da poliacrilamida $0,25 \%$.

4.13 Taxa de cisalhamento constante $-\dot{\gamma}=0,011 / s$ da goma xantana $0,40 \%$.

4.14 Curva de escoamento (Flow curve) da poliacrilamida 0,25\%.

4.15 Curva de escoamento (Flow curve) da goma xantana $0,40 \%$.

4.16 Curva de escoamento da poliacrilamida modelada por power law.

4.17 Curva de escoamento da goma xantana modelada por power law. $\quad 70$

4.18 Representação esquemática da resposta de um material tixotrópico. 72

4.19 Step change poliacrilamida $0,25 \%$.

4.20 Step change goma xantana $0,40 \%$.

4.21 Solução de glicerina $80 \%$ deslocando óleo mineral $-\mu^{*}=3,260$; $\rho^{*}=0,728 ; \dot{m}=2,180 \mathrm{~g} / \mathrm{s} ; W e=2,45 ; C a=5,76.10^{-4}$; $R e=1,98 ; T=22^{\circ} C ; \varepsilon=57,706 \%$; Fingers.

4.22 Solução de glicerina $80 \%$ deslocando óleo mineral $-\mu^{*}=3,395$; $\rho^{*}=0,728 ; \dot{m}=0,44 \mathrm{~g} / \mathrm{s} ; W e=0,098 ; C a=5,76.10^{-4}$; $R e=0,39 ; T=25^{\circ} C ; \varepsilon=54,863 \%$; Fingers.

4.23 Solução de glicerina $60 \%$ deslocando óleo mineral $-\mu^{*}=16,761$; $\rho^{*}=0,754 ; \dot{m}=0,367 \mathrm{~g} / \mathrm{s} ; W e=0,077 ; C a=2,62.10^{-4}$; $R e=0,81 ; T=22^{\circ} C ; \varepsilon=36,549 \%$; Fingers.

4.24 Óleo mineral deslocando solução de glicerina $80 \%-\mu^{*}=0,307$; $\rho^{*}=1,373 ; \dot{m}=2,561 \mathrm{~g} / \mathrm{s} ; W e=1,30 ; C a=3,07.10^{-3}$; $R e=0,23 ; T=22^{\circ} C ; \varepsilon=99,645 \%$; Plug.

4.25 Óleo mineral deslocando solução de glicerina $80 \%-\mu^{*}=0,302$; $\rho^{*}=1,373 ; \dot{m}=0,490 \mathrm{~g} / \mathrm{s} ; W e=0,049 ; C a=3,07.10^{-3}$; $R e=0,044 ; T=23^{\circ} \mathrm{C} ; \varepsilon=100,00 \%$; Plug.

4.26 Óleo mineral deslocando solução de glicerina $60 \%-\mu^{*}=0,056$; $\rho^{*}=1,373 ; \dot{m}=0,475 \mathrm{~g} / \mathrm{s} ; W e=0,551 ; C a=3,52.10^{-3}$; $R e=0,044 ; T=21^{\circ} C ; \varepsilon=100,00 \%$; Plug.

4.27 Óleo mineral deslocando ar $-\mu^{*}=1,37.10^{-4} ; \rho^{*}=0,0014$; $\dot{m}=0,344 \mathrm{~g} / \mathrm{s} ; W e=0,0478 ; C a=2,15.10^{-4} ; R e=0,042 ;$ $T=25^{\circ} \mathrm{C} \varepsilon=100,00 \%$, Plug.

4.28 Óleo mineral deslocando ar $-\mu^{*}=1,37.10^{-4} ; \rho^{*}=0,0014$; $\dot{m}=3,644 \mathrm{~g} / \mathrm{s} ; W e=5,346 ; C a=3,30 \cdot 10^{-3} ; R e=0,451$; $T=25^{\circ} \mathrm{C} \varepsilon=99,99 \%$, Plug. 
4.29 Óleo mineral deslocando solução de poliacrilamida $0,25 \%-\dot{\gamma}=$ $4,0001 / \mathrm{s} ; u^{*}=\dot{\gamma}^{*}=0,198 ; \eta^{*}=3,172 ; \dot{m}=0,021 \mathrm{~g} / \mathrm{s} ; R e=$ 0,$002 ; W e=1,299.10^{-6} ; C a=8,476.10^{-2} ; \mathrm{T}=20^{\circ} \mathrm{C} ; \varepsilon=$ 26, 832\%, Fingers.

4.30 Óleo mineral deslocando solução de poliacrilamida $0,25 \%-\dot{\gamma}=$ $10,8581 / \mathrm{s} ; u^{*}=\dot{\gamma}^{*}=0,538 ; \eta^{*}=1,555 ; \dot{m}=0,058 \mathrm{~g} / \mathrm{s} ;$ $R e=0,004 ; W e=9,571.10^{-6} ; C a=8,476.10^{-2} ; \mathrm{T}=20^{\circ} \mathrm{C}$; $\varepsilon=40,046 \%$, Fingers.

4.31 Óleo mineral deslocando solução de poliacrilamida $0,25 \%-\dot{\gamma}=$ $12,8761 / \mathrm{s} ; u^{*}=\dot{\gamma}^{*}=0,638 ; \eta^{*}=1,377 ; \dot{m}=0,069 \mathrm{~g} / \mathrm{s}$; $R e=0,005 ; W e=1,346.10^{-5} ; C a=8,476.10^{-2} ; \mathrm{T}=20^{\circ} \mathrm{C}$; $\varepsilon=39,376 \%$, Fingers.

4.32 Óleo mineral deslocando solução de poliacrilamida $0,25 \%-\dot{\gamma}=$ $18,9191 / \mathrm{s} ; u^{*}=\dot{\gamma}^{*}=0,777 ; \eta^{*}=1,198 ; \dot{m}=0,101 \mathrm{~g} / \mathrm{s}$; $R e=0,009 ; W e=2,906 \cdot 10^{-5} ; C a=8,850 \cdot 10^{-2} ; T=22^{\circ} C$; $\varepsilon=54,559 \%$, Fingers.

4.33 Óleo mineral deslocando solução de poliacrilamida $0,25 \%-\dot{\gamma}=$ $37,8591 / \mathrm{s} ; u^{*}=\dot{\gamma}^{*}=2,057 ; \eta^{*}=0,598 ; \dot{m}=0,202 \mathrm{~g} / \mathrm{s}$; $R e=0,014 ; W e=1,164.10^{-4} ; C a=8,301 \cdot 10^{-2} ; T=19^{\circ} C$; $\varepsilon=54,108 \%$, Fingers.

4.34 Óleo mineral deslocando solução de poliacrilamida $0,25 \%-\dot{\gamma}=$ $38,9911 / \mathrm{s} ; u^{*}=\dot{\gamma}^{*}=1,601 ; \eta^{*}=0,715 ; \dot{m}=0,208 \mathrm{~g} / \mathrm{s} ;$ $R e=0,018 ; W e=1,234.10^{-4} ; C a=8,850.10^{-2} ; T=22^{\circ} C$; $\varepsilon=64,416 \%$, Fingers.

4.35 Óleo mineral deslocando solução de poliacrilamida $0,25 \%-\dot{\gamma}=$ $42,6161 / \mathrm{s} ; u^{*}=\dot{\gamma}^{*}=2,113 ; \eta^{*}=0,586 ; \dot{m}=0,227 \mathrm{~g} / \mathrm{s}$; $R e=0,017 ; W e=1,474.10^{-4} ; C a=8,476.10^{-2} ; \mathrm{T}=20^{\circ} \mathrm{C}$; $\varepsilon=69,298 \%$, Fingers.

4.36 Óleo mineral deslocando solução de poliacrilamida $0,25 \%-\dot{\gamma}=$ $62,0971 / \mathrm{s} ; u^{*}=\dot{\gamma}^{*}=2,805 ; \eta^{*}=0,479 ; \dot{m}=0,331 \mathrm{~g} / \mathrm{s} ;$ $R e=0,026 ; W e=3,130.10^{-4} ; C a=8,659 \cdot 10^{-2} ; T=21^{\circ} \mathrm{C}$; $\varepsilon=77,717 \%$, Transição.

Óleo mineral deslocando solução de poliacrilamida $0,25 \%-\dot{\gamma}=$ $115,8241 / \mathrm{s} ; u^{*}=\dot{\gamma}^{*}=4,755 ; \eta^{*}=0,329 ; \dot{m}=0,618 \mathrm{~g} / \mathrm{s} ;$ $R e=0,052 ; W e=1,089.10^{-3} ; C a=8,850 \cdot 10^{-2} ; T=22^{\circ} C$; $\varepsilon=90,388 \%$, Transição.

4.38 Óleo mineral deslocando solução de poliacrilamida $0,25 \%-\dot{\gamma}=$ $175,3481 / \mathrm{s} ; u^{*}=\dot{\gamma}^{*}=7,198 ; \eta^{*}=0,245 ; \dot{m}=0,936 \mathrm{~g} / \mathrm{s} ;$ $R e=0,079 ; W e=2,496.10^{-3} ; C a=8,850.10^{-2} ; T=22^{\circ} C$; $\varepsilon=96,199 \%$, Plug.

4.39 Óleo mineral deslocando solução de poliacrilamida $0,25 \%-\dot{\gamma}=$ $283,7111 / \mathrm{s} ; u^{*}=\dot{\gamma}^{*}=11,646 ; \eta^{*}=0,173 ; \dot{m}=1,514 \mathrm{~g} / \mathrm{s}$; $R e=0,128 ; W e=6,535.10^{-3} ; C a=8,850.10^{-2} ; T=22^{\circ} C$; $\varepsilon=99,885 \%$, Plug.

4.40 Óleo mineral deslocando solução de poliacrilamida $0,25 \%-\dot{\gamma}=$ $380,6861 / \mathrm{s} ; u^{*}=\dot{\gamma}^{*}=15,627 ; \eta^{*}=0,141 ; \dot{m}=2,031 \mathrm{~g} / \mathrm{s}$; $R e=0,171 ; W e=1,177.10^{-2} ; C a=8,850.10^{-2} ; T=22^{\circ} C$; $\varepsilon=99,715 \%$, Plug. 
4.41 Óleo mineral deslocando solução de poliacrilamida $0,25 \%-\dot{\gamma}=$ 405, $0881 / \mathrm{s} ; u^{*}=\dot{\gamma}^{*}=16,629 ; \eta^{*}=0,135 ; \dot{m}=2,161 \mathrm{~g} / \mathrm{s}$; $R e=0,182 ; W e=1,332.10^{-2} ; C a=8,850.10^{-2} ; T=22^{\circ} C$; $\varepsilon=99,000 \%$, Plug.

4.42 Óleo mineral deslocando solução de poliacrilamida $0,25 \%-\dot{\gamma}=$ $462,8621 / \mathrm{s} ; u^{*}=\dot{\gamma}^{*}=22,952 ; \eta^{*}=0,107 ; \dot{m}=2,470 \mathrm{~g} / \mathrm{s}$; $R e=0,182 ; W e=1,739.10^{-2} ; C a=8,476.10^{-2} ; T=20^{\circ} C$; $\varepsilon=99,526 \%$, Plug.

4.43 Óleo mineral deslocando solução de poliacrilamida $0,25 \%-\dot{\gamma}=$ 487,003 1/s; $u^{*}=\dot{\gamma}^{*}=21,996 ; \eta^{*}=0,110 ; \dot{m}=2,598 \mathrm{~g} / \mathrm{s}$; $R e=0,205 ; W e=1,925.10^{-2} ; C a=8,659.10^{-2} ; T=21^{\circ} C$; $\varepsilon=98,513 \%$, Plug.

4.44 Óleo mineral deslocando solução de poliacrilamida $0,25 \%-\dot{\gamma}=$ $562,2691 / \mathrm{s} ; u^{*}=\dot{\gamma}^{*}=23,081 ; \eta^{*}=0,106 ; \dot{m}=3,000 \mathrm{~g} / \mathrm{s}$; $R e=0,253 ; W e=2,567.10^{-2} ; C a=8,850.10^{-2} ; T=22^{\circ} C$; $\varepsilon=99,799 \%$, Plug.

4.45 Óleo mineral deslocando solução de poliacrilamida $0,25 \%-\dot{\gamma}=$ $652,8571 / \mathrm{s} ; u^{*}=\dot{\gamma}^{*}=26,799 ; \eta^{*}=0,096 ; \dot{m}=3,483 \mathrm{~g} / \mathrm{s}$; $R e=0,294 ; W e=3,460.10^{-2} ; C a=8,850.10^{-2} ; \mathrm{T}=22^{\circ} \mathrm{C}$; $\varepsilon=99,874 \%$, Plug.

4.46 Óleo mineral deslocando solução de poliacrilamida $0,25 \%-\dot{\gamma}=$ $805,9191 / \mathrm{s} ; u^{*}=\dot{\gamma}^{*}=33,083 ; \eta^{*}=0,082 ; \dot{m}=4,300 \mathrm{~g} / \mathrm{s}$; $R e=0,362 ; W e=5,273.10^{-2} ; C a=8,850.10^{-2} ; T=22^{\circ} C$; $\varepsilon=99,757 \%$, Plug.

4.47 Óleo deslocando solução de poliacrilamida - Reynolds em função da velocidade.

4.48 Óleo deslocando solução de poliacrilamida - Eficiência em função da velocidade adimensional.

4.49 Óleo deslocando solução de poliacrilamida - Eficiência em função da viscosidade adimensional.

4.50 Óleo deslocando solução de poliacrilamida - Viscosidade adimensional em função da taxa de cisalhamento adimensional.

4.51 Óleo deslocando solução de poliacrilamida - Padrões de escoamento, fingers e plug.

4.52 Solução de poliacrilamida $0,25 \%$ deslocando óleo mineral $-\dot{\gamma}=$ $4,7461 / \mathrm{s} ; u^{*}=\dot{\gamma}^{*}=0,160 ; \eta^{*}=3,699 ; \dot{m}=0,022 \mathrm{~g} / \mathrm{s}$; $R e=0,0008 ; W e=2,100.10^{-9} ; C a=3,425.10^{-1} ; T=24^{\circ} C$; $\varepsilon=100 \%$, Plug.

4.53 Solução de poliacrilamida $0,25 \%$ deslocando óleo mineral $-\dot{\gamma}=$ $14,7911 / \mathrm{s} ; u^{*}=\dot{\gamma}^{*}=0,578 ; \eta^{*}=1,478 ; \dot{m}=0,069 \mathrm{~g} / \mathrm{s}$; $R e=0,005 ; W e=2,040.10^{-8} ; C a=1,323.10^{-1} ; T=22,5^{\circ} \mathrm{C}$; $\varepsilon=99,984 \%$, Plug.

4.54 Solução de poliacrilamida $0,25 \%$ deslocando óleo mineral $-\dot{\gamma}=$ $33,5011 / \mathrm{s} ; u^{*}=\dot{\gamma}^{*}=1,129 ; \eta^{*}=0,917 ; \dot{m}=0,156 \mathrm{~g} / \mathrm{s} ;$ $R e=0,022 ; W e=1,046.10^{-7} ; C a=8,494.10^{-2} ; T=24^{\circ} C ; \varepsilon=$ 98, 940\%, Plug. 
4.55 Solução de poliacrilamida $0,25 \%$ deslocando óleo mineral $-\dot{\gamma}=$ $36,8881 / \mathrm{s} ; u^{*}=\dot{\gamma}^{*}=1,414 ; \eta^{*}=0,781 ; \dot{m}=0,171 \mathrm{~g} / \mathrm{s} ;$ $R e=0,026 ; W e=1,269.10^{-7} ; C a=7,019.10^{-2} ; T=22,7^{\circ} C$; $\varepsilon=99,751 \%$, Plug.

4.56 Solução de poliacrilamida $0,25 \%$ deslocando óleo mineral $-\dot{\gamma}=$ $42,9321 / \mathrm{s} ; u^{*}=\dot{\gamma}^{*}=1,646 ; \eta^{*}=0,701 ; \dot{m}=0,199 \mathrm{~g} / \mathrm{s}$; $R e=0,033 ; W e=1,719.10^{-7} ; C a=6,299.10^{-2} ; T=22,7^{\circ} C$; $\varepsilon=99,734 \%$, Plug.

4.57 Solução de poliacrilamida $0,25 \%$ deslocando óleo mineral $-\dot{\gamma}=$ $47,1111 / \mathrm{s} ; u^{*}=\dot{\gamma}^{*}=1,824 ; \eta^{*}=0,651 ; \dot{m}=0,219 \mathrm{~g} / \mathrm{s}$; $R e=0,039 ; W e=2,069.10^{-7} ; C a=5,841.10^{-2} ; T=$ $22,6^{\circ} \mathrm{C} ; \varepsilon=98,956 \%$, Plug.

4.58 Solução de poliacrilamida $0,25 \%$ deslocando óleo mineral $-\dot{\gamma}=$ $59,2641 / \mathrm{s} ; u^{*}=\dot{\gamma}^{*}=2,295 ; \eta^{*}=0,553 ; \dot{m}=0,275 \mathrm{~g} / \mathrm{s} ;$ $R e=0,058 ; W e=3,270.10^{-7} ; C a=4,959.10^{-2} ; T=$ $22,6^{\circ} \mathrm{C} ; \varepsilon=96,888 \%$, Plug.

4.59 Solução de poliacrilamida $0,25 \%$ deslocando óleo mineral $-\dot{\gamma}=$ $64,431 / \mathrm{s} ; u^{*}=\dot{\gamma}^{*}=2,170 ; \eta^{*}=0,575 ; \dot{m}=0,299 \mathrm{~g} / \mathrm{s}$; $R e=0,067 ; W e=3,870 \cdot 10^{-7} ; C a=5,327.10^{-2} ; T=24^{\circ} C ; \varepsilon=$ 94, $03 \%$, Plug.

4.60 Solução de poliacrilamida $0,25 \%$ deslocando óleo mineral $-\dot{\gamma}=$ $82,1471 / \mathrm{s} ; u^{*}=\dot{\gamma}^{*}=3,150 ; \eta^{*}=0,441 ; \dot{m}=0,382 \mathrm{~g} / \mathrm{s} ;$ $R e=0,101 ; W e=6,292.10^{-7} ; C a=3,965 \cdot 10^{-2} ; T=$ $22,7^{\circ} \mathrm{C} ; \varepsilon=89,899 \%$, Transição.

4.61 Solução de poliacrilamida $0,25 \%$ deslocando óleo mineral $-\dot{\gamma}=$ $98,0931 / \mathrm{s} ; u^{*}=\dot{\gamma}^{*}=3,304 ; \eta^{*}=0,426 ; \dot{m}=0,456 \mathrm{~g} / \mathrm{s}$; $R e=0,137 ; W e=8,972.10^{-7} ; C a=3,946 \cdot 10^{-2} ; T=24^{\circ} \mathrm{C}$; $\varepsilon=77,100 \%$, Transição.

4.62 Solução de poliacrilamida $0,25 \%$ deslocando óleo mineral $-\dot{\gamma}=$ $132,4221 / \mathrm{s} ; u^{*}=\dot{\gamma}^{*}=4,930 ; \eta^{*}=0,320 ; \dot{m}=0,615 \mathrm{~g} / \mathrm{s} ;$ $R e=0,229 ; W e=1,635.10^{-6} ; C a=2,900.10^{-2} ; T=23^{\circ} C ; \varepsilon=$ $71,760 \%$, Fingers.

4.63 Solução de poliacrilamida $0,25 \%$ deslocando óleo mineral $-\dot{\gamma}=$ $157,1361 / \mathrm{s} ; u^{*}=\dot{\gamma}^{*}=5,850 ; \eta^{*}=0,284 ; \dot{m}=0,730 \mathrm{~g} / \mathrm{s} ;$ $R e=0,307 ; W e=2,302 \cdot 10^{-6} ; C a=2,566.10^{-2} ; T=23^{\circ} C ; \varepsilon=$ $61,770 \%$, Fingers.

4.64 Solução de poliacrilamida $0,25 \%$ deslocando óleo mineral $-\dot{\gamma}=$ $186,5201 / \mathrm{s} ; u^{*}=\dot{\gamma}^{*}=6,944 ; \eta^{*}=0,251 ; \dot{m}=0,867 \mathrm{~g} / \mathrm{s}$; $R e=0,412 ; W e=3,244.10^{-6} ; C a=2,271.10^{-2} ; T=23^{\circ} C ; \varepsilon=$ $65,960 \%$, Fingers.

4.65 Solução de poliacrilamida $0,25 \%$ deslocando óleo mineral $-\dot{\gamma}=$ $228,3481 / \mathrm{s} ; u^{*}=\dot{\gamma}^{*}=9,374 ; \eta^{*}=0,203 ; \dot{m}=1,061 \mathrm{~g} / \mathrm{s}$; $R e=0,582 ; W e=4,862.10^{-6} ; C a=1,793.10^{-2} ; T=22^{\circ} C ; \varepsilon=$ $55,940 \%$, Fingers.

4.66 Solução de poliacrilamida $0,25 \%$ deslocando óleo mineral $-\dot{\gamma}=$ $272,8951 / \mathrm{s} ; u^{*}=\dot{\gamma}^{*}=12,326 ; \eta^{*}=0,167 ; \dot{m}=1,268 \mathrm{~g} / \mathrm{s}$; $R e=0,790 ; W e=6,943.10^{-6} ; C a=1,443.10^{-2} ; T=21^{\circ} C ; \varepsilon=$ $33,620 \%$, Fingers. 
4.67 Solução de poliacrilamida $0,25 \%$ deslocando óleo mineral $-\dot{\gamma}=$ $303,9781 / \mathrm{s} ; u^{*}=\dot{\gamma}^{*}=12,48 ; \eta^{*}=0,165 ; \dot{m}=1,412 \mathrm{~g} / \mathrm{s}$; $R e=0,951 ; W e=8,615.10^{-6} ; C a=1,462.10^{-2} ; T=22^{\circ} C ; \varepsilon=$ 63, $940 \%$, Fingers.

4.68 Solução de poliacrilamida $0,25 \%$ deslocando óleo mineral $-\dot{\gamma}=$ $311,3391 / \mathrm{s} ; u^{*}=\dot{\gamma}^{*}=14,062 ; \eta^{*}=0,152 ; \dot{m}=1,446 \mathrm{~g} / \mathrm{s}$; $R e=0,990 ; W e=9,039.10^{-6} ; C a=1,313.10^{-2} ; T=21^{\circ} C ; \varepsilon=$ $63,940 \%$, Fingers.

4.69 Solução de poliacrilamida $0,25 \%$ deslocando óleo mineral $-\dot{\gamma}=$ $355,4471 / \mathrm{s} ; u^{*}=\dot{\gamma}^{*}=14,591 ; \eta^{*}=0,148 ; \dot{m}=1,651 \mathrm{~g} / \mathrm{s} ;$ $R e=1,243 ; W e=1,178 \cdot 10^{-5} ; C a=1,307.10^{-2} ; T=22^{\circ} C ; \varepsilon=$ $56,198 \%$, Fingers.

4.70 Solução de poliacrilamida $0,25 \%$ deslocando óleo mineral $-\dot{\gamma}=$ $410,9721 / \mathrm{s} ; u^{*}=\dot{\gamma}^{*}=16,870 ; \eta^{*}=0,133 ; \dot{m}=1,909 \mathrm{~g} / \mathrm{s} ;$ $R e=1,594 ; W e=1,575.10^{-5} ; C a=1,179.10^{-2} ; T=22^{\circ} C$; $\varepsilon=61,920 \%$, Fingers.

4.71 Solução de poliacrilamida $0,25 \%$ deslocando óleo mineral $-\dot{\gamma}=$ $478,5131 / \mathrm{s} ; u^{*}=\dot{\gamma}^{*}=19,643 ; \eta^{*}=0,119 ; \dot{m}=2,223 \mathrm{~g} / \mathrm{s} ;$ $R e=2,068 ; W e=2,135.10^{-5} ; C a=1,057.10^{-2} ; T=22^{\circ} C ; \varepsilon=$ $41,143 \%$, Fingers.

4.72 Solução de poliacrilamida deslocando óleo - Reynolds em função da velocidade.

4.73 Solução de poliacrilamida deslocando óleo - Eficiência em função da velocidade adimensional.

4.74 Solução de poliacrilamida deslocando óleo - Eficiência em função da viscosidade adimensional.

4.75 Solução de poliacrilamida deslocando óleo - Viscosidade adimensional em função da taxa de cisalhamento adimensional.

4.76 Solução de poliacrilamida deslocando óleo - Padrões de escoamento, fingers e plug.

4.77 Óleo mineral deslocando solução de goma xantana $0,40 \%-\dot{\gamma}=$ $10,6501 / \mathrm{s} ; u^{*}=\dot{\gamma}^{*}=0,467 ; \eta^{*}=1,760 ; \dot{m}=0,052 \mathrm{~g} / \mathrm{s} ;$ $R e=0,005 ; W e=8,160.10^{-6} ; C a=8,545.10^{-2} ; T=23^{\circ} \mathrm{C}$; $\varepsilon=31,023 \%$, Fingers.

4.78 Óleo mineral deslocando solução de goma xantana $0,40 \%-\dot{\gamma}=$ $16,3071 / \mathrm{s} ; u^{*}=\dot{\gamma}^{*}=0,816 ; \eta^{*}=1,163 ; \dot{m}=0,080 \mathrm{~g} / \mathrm{s} ;$ $R e=0,007 ; W e=1,913.10^{-5} ; C a=8,263.10^{-2} ; \mathrm{T}=22^{\circ} \mathrm{C}$; $\varepsilon=47,154 \%$, Fingers.

4.79 Óleo mineral deslocando solução de goma xantana $0,40 \%-\dot{\gamma}=$ $18,5851 / \mathrm{s} ; u^{*}=\dot{\gamma}^{*}=0,816 ; \eta^{*}=1,164 ; \dot{m}=0,092 \mathrm{~g} / \mathrm{s}$; $R e=0,008 ; W e=2,485.10^{-5} ; C a=8,545.10^{-2} ; T=23^{\circ} C ; \varepsilon=$ $39,267 \%$, Fingers.

4.80 Óleo mineral deslocando solução de goma xantana $0,40 \%-\dot{\gamma}=$ $32,0441 / \mathrm{s} ; u^{*}=\dot{\gamma}^{*}=1,406 ; \eta^{*}=0,776 ; \dot{m}=0,158 \mathrm{~g} / \mathrm{s} ;$ $R e=0,014 ; W e=7,386.10^{-5} ; C a=8,545.10^{-2} ; \mathrm{T}=23^{\circ} \mathrm{C}$; $\varepsilon=41,865 \%$, Fingers. 
4.81 Óleo mineral deslocando solução de goma xantana $0,40 \%-\dot{\gamma}=$ $34,5991 / \mathrm{s} ; u^{*}=\dot{\gamma}^{*}=1,518 ; \eta^{*}=0,733 ; \dot{m}=0,171 \mathrm{~g} / \mathrm{s}$; $R e=0,016 ; W e=8,612.10^{-5} ; C a=8,545.10^{-2} ; \mathrm{T}=23^{\circ} \mathrm{C}$; $\varepsilon=58,836 \%$, Fingers.

4.82 Óleo mineral deslocando solução de goma xantana $0,40 \%-\dot{\gamma}=$ $34,8281 / \mathrm{s} ; u^{*}=\dot{\gamma}^{*}=1,679 ; \eta^{*}=0,681 ; \dot{m}=0,172 \mathrm{~g} / \mathrm{s}$; $R e=0,015 ; W e=8,726.10^{-5} ; C a=8,342.10^{-2} ; T=22^{\circ} C$; $\varepsilon=50,764 \%$, Fingers.

4.83 Óleo mineral deslocando solução de goma xantana $0,40 \%-\dot{\gamma}=$ $38,7171 / \mathrm{s} ; u^{*}=\dot{\gamma}^{*}=2,238 ; \eta^{*}=0,550 ; \dot{m}=0,191 \mathrm{~g} / \mathrm{s}$; $R e=0,014 ; W e=1,078.10^{-4} ; C a=7,962.10^{-2} ; \mathrm{T}=20^{\circ} \mathrm{C}$; $\varepsilon=67,772 \%$, Fingers.

4.84 Óleo mineral deslocando solução de goma xantana $0,40 \%-\dot{\gamma}=$ $53,2611 / \mathrm{s} ; u^{*}=\dot{\gamma}^{*}=2,427 ; \eta^{*}=0,517 ; \dot{m}=0,263 \mathrm{~g} / \mathrm{s} ;$ $R e=0,023 ; W e=2,041.10^{-4} ; C a=8,463.10^{-2} ; T=23^{\circ} \mathrm{C}$; $\varepsilon=76,563 \%$, Fingers.

4.85 Óleo mineral deslocando solução de goma xantana $0,40 \%-\dot{\gamma}=$ $53,2851 / \mathrm{s} ; u^{*}=\dot{\gamma}^{*}=2,616 ; \eta^{*}=0,489 ; \dot{m}=0,263 \mathrm{~g} / \mathrm{s} ;$ $R e=0,022 ; W e=2,042.10^{-4} ; C a=8,302.10^{-2} ; \mathrm{T}=22^{\circ} \mathrm{C}$; $\varepsilon=76,037 \%$, Fingers.

4.86 Óleo mineral deslocando solução de goma xantana $0,40 \%-\dot{\gamma}=$ $58,7811 / \mathrm{s} ; u^{*}=\dot{\gamma}^{*}=2,755 ; \eta^{*}=0,471 ; \dot{m}=0,290 \mathrm{~g} / \mathrm{s} ;$ $R e=0,025 ; W e=2,486.10^{-4} ; C a=8,402.10^{-2} ; T=22^{\circ} C$; $\varepsilon=80,178 \%$, Fingers.

4.87 Óleo mineral deslocando solução de goma xantana $0,40 \%-\dot{\gamma}=$ $63,4961 / \mathrm{s} ; u^{*}=\dot{\gamma}^{*}=3,056 ; \eta^{*}=0,436 ; \dot{m}=0,313 \mathrm{~g} / \mathrm{s}$; $R e=0,027 ; W e=2,891.10^{-4} ; C a=8,342.10^{-2} ; T=22^{\circ} C$; $\varepsilon=81,234 \%$, Fingers.

4.88 Óleo mineral deslocando solução de goma xantana $0,40 \%-\dot{\gamma}=$ $63,5291 / \mathrm{s} ; u^{*}=\dot{\gamma}^{*}=3,387 ; \eta^{*}=0,404 ; \dot{m}=0,313 \mathrm{~g} / \mathrm{s} ;$ $R e=0,025 ; W e=2,903.10^{-4} ; C a=8,128 \cdot 10^{-2} ; T=21^{\circ} \mathrm{C}$; $\varepsilon=85,118 \%$, Transição.

4.89 Óleo mineral deslocando solução de goma xantana $0,40 \%-\dot{\gamma}=$ $68,6331 / \mathrm{s} ; u^{*}=\dot{\gamma}^{*}=3,217 ; \eta^{*}=0,420 ; \dot{m}=0,338 \mathrm{~g} / \mathrm{s}$; $R e=0,029 ; W e=3,389.10^{-4} ; C a=8,402.10^{-2} ; T=22^{\circ} C$; $\varepsilon=82,483 \%$, Transição.

4.90 Óleo mineral deslocando solução de goma xantana $0,40 \%-\dot{\gamma}=$ $74,1681 / \mathrm{s} ; u^{*}=\dot{\gamma}^{*}=3,380 ; \eta^{*}=0,405 ; \dot{m}=0,366 \mathrm{~g} / \mathrm{s} ;$ $R e=0,032 ; W e=3,957.10^{-4} ; C a=8,463.10^{-2} ; T=23^{\circ} \mathrm{C}$; $\varepsilon=89,019 \%$, Transição.

4.91 Óleo mineral deslocando solução de goma xantana $0,40 \%-\dot{\gamma}=$ $197,7141 / \mathrm{s} ; u^{*}=\dot{\gamma}^{*}=8,676 ; \eta^{*}=0,201 ; \dot{m}=0,975 \mathrm{~g} / \mathrm{s}$; $R e=0,089 ; W e=2,812.10^{-3} ; C a=8,545.10^{-2} ; T=23^{\circ} \mathrm{C}$; $\varepsilon=99,931 \%$, Plug.

4.92 Óleo mineral deslocando solução de goma xantana $0,40 \%-\dot{\gamma}=$ $229,3651 / \mathrm{s} ; u^{*}=\dot{\gamma}^{*}=9,596 ; \eta^{*}=0,186 ; \dot{m}=1,131 \mathrm{~g} / \mathrm{s}$; $R e=0,107 ; W e=3,784.10^{-3} ; C a=8,651.10^{-2} ; T=24^{\circ} C$; $\varepsilon=99,696 \%$, Plug. 
4.93 Óleo mineral deslocando solução de goma xantana $0,40 \%-\dot{\gamma}=$ $249,6211 / \mathrm{s} ; u^{*}=\dot{\gamma}^{*}=9,952 ; \eta^{*}=0,181 ; \dot{m}=1,231 \mathrm{~g} / \mathrm{s} ;$ $R e=0,120 ; W e=4,482.10^{-3} ; C a=8,759.10^{-2} ; T=24^{\circ} \mathrm{C}$; $\varepsilon=100 \%$, Plug.

4.94 Óleo mineral deslocando solução de goma xantana $0,40 \%-\dot{\gamma}=$ $254,0121 / \mathrm{s} ; u^{*}=\dot{\gamma}^{*}=11,360 ; \eta^{*}=0,164 ; \dot{m}=1,252 \mathrm{~g} / \mathrm{s}$; $R e=0,113 ; W e=4,642.10^{-3} ; C a=8,504 \cdot 10^{-2} ; T=23^{\circ} C$; $\varepsilon=98,434 \%$, Plug.

4.95 Óleo mineral deslocando solução de goma xantana $0,40 \%-\dot{\gamma}=$ $275,3041 / \mathrm{s} ; u^{*}=\dot{\gamma}^{*}=10,976 ; \eta^{*}=0,169 ; \dot{m}=1,357 \mathrm{~g} / \mathrm{s}$; $R e=0,133 ; W e=5,452.10^{-3} ; C a=8,759.10^{-2} ; T=24^{\circ} C$; $\varepsilon=99,919 \%$, Plug.

4.96 Óleo mineral deslocando solução de goma xantana $0,40 \%-\dot{\gamma}=$ $282,7361 / \mathrm{s} ; u^{*}=\dot{\gamma}^{*}=13,629 ; \eta^{*}=0,144 ; \dot{m}=1,394 \mathrm{~g} / \mathrm{s} ;$ $R e=0,118 ; W e=5,751.10^{-3} ; C a=8,342.10^{-2} ; \mathrm{T}=22^{\circ} \mathrm{C}$; $\varepsilon=99,908 \%$, Plug.

4.97 Óleo mineral deslocando solução de goma xantana $0,40 \%-\dot{\gamma}=$ $313,6141 / \mathrm{s} ; u^{*}=\dot{\gamma}^{*}=14,564 ; \eta^{*}=0,137 ; \dot{m}=1,546 \mathrm{~g} / \mathrm{s}$; $R e=0,135 ; W e=7,075.10^{-3} ; C a=8,422.10^{-2} ; T=22^{\circ} C$; $\varepsilon=99,319 \%$, Plug.

4.98 Óleo deslocando solução de goma xantana - Reynolds em função da velocidade.

4.99 Óleo deslocando solução de goma xantana - Eficiência em função da velocidade adimensional.

4.100Óleo deslocando solução de goma xantana - Eficiência em função da viscosidade adimensional.

4.101Óleo deslocando solução de goma xantana - Viscosidade adimensional em função da taxa de cisalhamento adimensional.

4.102Óleo deslocando solução de goma xantana - Padrões de escoamento, fingers e plug.

4.103Solução de goma xantana $0,40 \%$ deslocando óleo mineral $-\dot{\gamma}=$ $7,8861 / \mathrm{s} ; u^{*}=\dot{\gamma}^{*}=0,314 ; \eta^{*}=2,362 ; \dot{m}=0,034 \mathrm{~g} / \mathrm{s}$; $R e=0,004 ; W e=5,095.10^{-6} ; C a=2,069.10^{-1} ; T=24^{\circ} C$; $\varepsilon=99,454 \%$, Plug.

4.104Solução de goma xantana $0,40 \%$ deslocando óleo mineral $-\dot{\gamma}=$ $11,1831 / \mathrm{s} ; u^{*}=\dot{\gamma}^{*}=0,446 ; \eta^{*}=1,822 ; \dot{m}=0,048 \mathrm{~g} / \mathrm{s}$; $R e=0,003 ; W e=1,025.10^{-5} ; C a=1,596.10^{-1} ; T=24^{\circ} C$; $\varepsilon=99,876 \%$, Plug.

4.105Solução de goma xantana $0,40 \%$ deslocando óleo mineral $-\dot{\gamma}=$ $12,4771 / \mathrm{s} ; u^{*}=\dot{\gamma}^{*}=0,408 ; \eta^{*}=1,946 ; \dot{m}=0,054 \mathrm{~g} / \mathrm{s} ;$ $R e=0,004 ; W e=1,275.10^{-5} ; C a=1,793.10^{-1} ; T=26^{\circ} \mathrm{C}$; $\varepsilon=99,293 \%$, Plug.

4.106Solução de goma xantana $0,40 \%$ deslocando óleo mineral $-\dot{\gamma}=$ $12,5471 / \mathrm{s} ; u^{*}=\dot{\gamma}^{*}=0,410 ; \eta^{*}=1,938 ; \dot{m}=0,054 \mathrm{~g} / \mathrm{s} ;$ $R e=0,004 ; W e=1,290.10^{-5} ; C a=1,786.10^{-1} ; T=26^{\circ} C$; $\varepsilon=97,472 \%$, Plug. 
4.107Solução de goma xantana $0,40 \%$ deslocando óleo mineral $-\dot{\gamma}=$ $21,3681 / \mathrm{s} ; u^{*}=\dot{\gamma}^{*}=0,772 ; \eta^{*}=1,121 ; \dot{m}=0,093 \mathrm{~g} / \mathrm{s}$; $R e=0,010 ; W e=3,740.10^{-5} ; C a=1,088.10^{-1} ; T=25^{\circ} C$; $\varepsilon=99,347 \%$, Plug.

4.108Solução de goma xantana $0,40 \%$ deslocando óleo mineral $-\dot{\gamma}=$ $23,2811 / \mathrm{s} ; u^{*}=\dot{\gamma}^{*}=0,842 ; \eta^{*}=1,137 ; \dot{m}=0,101 \mathrm{~g} / \mathrm{s}$; $R e=0,012 ; W e=4,440.10^{-5} ; C a=1,021.10^{-1} ; T=25^{\circ} C$; $\varepsilon=98,011 \%$, Plug.

4.109Solução de goma xantana $0,40 \%$ deslocando óleo mineral $-\dot{\gamma}=$ $33,6291 / \mathrm{s} ; u^{*}=\dot{\gamma}^{*}=1,341 ; \eta^{*}=0,804 ; \dot{m}=0,146 \mathrm{~g} / \mathrm{s}$; $R e=0,023 ; W e=9,265.10^{-5} ; C a=7,044.10^{-2} ; \mathrm{T}=24^{\circ} \mathrm{C}$; $\varepsilon=98,291 \%$, Plug.

4.110Solução de goma xantana $0,40 \%$ deslocando óleo mineral $-\dot{\gamma}=$ $42,3381 / \mathrm{s} ; u^{*}=\dot{\gamma}^{*}=1,688 ; \eta^{*}=0,678 ; \dot{m}=0,183 \mathrm{~g} / \mathrm{s}$; $R e=0,034 ; W e=1,468.10^{-4} ; C a=5,936.10^{-2} ; T=24^{\circ} C$; $\varepsilon=95,365 \%$, Plug.

4.111Solução de goma xantana $0,40 \%$ deslocando óleo mineral $-\dot{\gamma}=$ $56,7871 / \mathrm{s} ; u^{*}=\dot{\gamma}^{*}=2,264 ; \eta^{*}=0,545 ; \dot{m}=0,246 \mathrm{~g} / \mathrm{s}$; $R e=0,034 ; W e=2,642.10^{-4} ; C a=4,773.10^{-2} ; \mathrm{T}=24^{\circ} \mathrm{C}$; $\varepsilon=83,656 \%$, Transição.

4.112Solução de goma xantana $0,40 \%$ deslocando óleo mineral $-\dot{\gamma}=$ 64, $0771 / \mathrm{s} ; u^{*}=\dot{\gamma}^{*}=2,555 ; \eta^{*}=0,498 ; \dot{m}=0,277 \mathrm{~g} / \mathrm{s}$; $R e=0,071 ; W e=3,363.10^{-4} ; C a=4,363.10^{-2} ; \mathrm{T}=24^{\circ} \mathrm{C}$; $\varepsilon=75,448 \%$, Fingers.

4.113Solução de goma xantana $0,40 \%$ deslocando óleo mineral $-\dot{\gamma}=$ $74,2181 / \mathrm{s} ; u^{*}=\dot{\gamma}^{*}=2,959 ; \eta^{*}=0,447 ; \dot{m}=0,321 \mathrm{~g} / \mathrm{s}$; $R e=0,091 ; W e=4,512.10^{-4} ; C a=3,912.10^{-2} ; T=24^{\circ} C$; $\varepsilon=53,451 \%$, Fingers.

4.114Solução de goma xantana $0,40 \%$ deslocando óleo mineral $-\dot{\gamma}=$ $80,8031 / \mathrm{s} ; u^{*}=\dot{\gamma}^{*}=3,222 ; \eta^{*}=0,419 ; \dot{m}=0,350 \mathrm{~g} / \mathrm{s}$; $R e=0,106 ; W e=5,349.10^{-4} ; C a=3,672.10^{-2} ; T=24^{\circ} \mathrm{C}$; $\varepsilon=65,525 \%$, Fingers.

4.115Solução de goma xantana $0,40 \%$ deslocando óleo mineral $-\dot{\gamma}=$ $107,9041 / \mathrm{s} ; u^{*}=\dot{\gamma}^{*}=4,302 ; \eta^{*}=0,338 ; \dot{m}=0,467 \mathrm{~g} / \mathrm{s}$; $R e=0,175 ; W e=9,538.10^{-4} ; C a=2,962 \cdot 10^{-2} ; T=24^{\circ} C$; $\varepsilon=45,537 \%$, Fingers.

4.116Solução de goma xantana $0,40 \%$ deslocando óleo mineral $-\dot{\gamma}=$ $136,4171 / \mathrm{s} ; u^{*}=\dot{\gamma}^{*}=5,439 ; \eta^{*}=0,384 ; \dot{m}=0,591 \mathrm{~g} / \mathrm{s}$; $R e=0,264 ; W e=1,524.10^{-3} ; C a=2,489.10^{-2} ; T=24^{\circ} C$; $\varepsilon=37,168 \%$, Fingers.

4.117Solução de goma xantana $0,40 \%$ deslocando óleo mineral $-\dot{\gamma}=$ $145,2491 / \mathrm{s} ; u^{*}=\dot{\gamma}^{*}=5,790 ; \eta^{*}=0,271 ; \dot{m}=0,629 \mathrm{~g} / \mathrm{s}$; $R e=0,294 ; W e=1,728.10^{-3} ; C a=2,375.10^{-2} ; T=24^{\circ} C$; $\varepsilon=32,983 \%$, Fingers.

4.118Solução de goma xantana $0,40 \%$ deslocando óleo mineral $-\dot{\gamma}=$ $164,2461 / \mathrm{s} ; u^{*}=\dot{\gamma}^{*}=7,208 ; \eta^{*}=0,230 ; \dot{m}=0,711 \mathrm{~g} / \mathrm{s}$; $R e=0,365 ; W e=2,210.10^{-3} ; C a=1,970.10^{-2} ; T=23^{\circ} \mathrm{C}$; $\varepsilon=37,218 \%$, Fingers. 
4.119Solução de goma xantana $0,40 \%$ deslocando óleo mineral $-\dot{\gamma}=$ $179,4661 / \mathrm{s} ; u^{*}=\dot{\gamma}^{*}=7,15 ; \eta^{*}=0,232 ; \dot{m}=0,777 \mathrm{~g} / \mathrm{s}$; $R e=0,426 ; W e=2,638.10^{-3} ; C a=2,030.10^{-2} ; T=24^{\circ} C$; $\varepsilon=32,940 \%$, Fingers.

4.120Solução de goma xantana $0,40 \%$ deslocando óleo mineral $-\dot{\gamma}=$ $187,8321 / \mathrm{s} ; u^{*}=\dot{\gamma}^{*}=7,489 ; \eta^{*}=0,224 ; \dot{m}=0,813 \mathrm{~g} / \mathrm{s}$; $R e=0,461 ; W e=2,890.10^{-3} ; C a=1,962.10^{-2} ; T=24^{\circ} C$; $\varepsilon=44,523 \%$, Fingers.

4.121Solução de goma xantana $0,40 \%$ deslocando óleo mineral $-\dot{\gamma}=$ $207,8251 / \mathrm{s} ; u^{*}=\dot{\gamma}^{*}=8,286 ; \eta^{*}=0,208 ; \dot{m}=0,900 \mathrm{~g} / \mathrm{s}$; $R e=0,550 ; W e=3,538.10^{-3} ; C a=1,820 \cdot 10^{-2} ; T=24^{\circ} \mathrm{C}$; $\varepsilon=30,436 \%$, Fingers.

4.122Solução de goma xantana $0,40 \%$ deslocando óleo mineral $-\dot{\gamma}=$ 240, $191 / \mathrm{s} ; u^{*}=\dot{\gamma}^{*}=9,576 ; \eta^{*}=0,187 ; \dot{m}=1,040 \mathrm{~g} / \mathrm{s}$; $R e=0,707 ; W e=4,726.10^{-3} ; C a=1,635.10^{-2} ; T=24^{\circ} C$; $\varepsilon=28,370 \%$, Fingers.

4.123Solução de goma xantana $0,40 \%$ deslocando óleo mineral $-\dot{\gamma}=$ 276,971 1/s; $u^{*}=\dot{\gamma}^{*}=11,043 ; \eta^{*}=0,168 ; \dot{m}=1,199 \mathrm{~g} / \mathrm{s} ;$ $R e=0,907 ; W e=6,284.10^{-3} ; C a=1,479.10^{-2} ; T=24^{\circ} C$; $\varepsilon=25,609 \%$, Fingers.

4.124Solução de goma xantana $0,40 \%$ deslocando óleo mineral $-\dot{\gamma}=$ $291,3101 / \mathrm{s} ; u^{*}=\dot{\gamma}^{*}=12,784 ; \eta^{*}=0,151 ; \dot{m}=1,261 \mathrm{~g} / \mathrm{s}$; $R e=0,990 ; W e=6,952.10^{-3} ; C a=1,287.10^{-2} ; T=23^{\circ} C$; $\varepsilon=31,109 \%$, Fingers.

4.125Solução de goma xantana $0,40 \%$ deslocando óleo mineral $-\dot{\gamma}=$ $326,3641 / \mathrm{s} ; u^{*}=\dot{\gamma}^{*}=14,322 ; \eta^{*}=0,138 ; \dot{m}=1,413 \mathrm{~g} / \mathrm{s}$; $R e=1,207 ; W e=8,726.10^{-3} ; C a=1,183.10^{-2} ; \mathrm{T}=23^{\circ} \mathrm{C}$; $\varepsilon=23,568 \%$, Fingers.

4.126Solução de goma xantana deslocando óleo - Reynolds em função da velocidade.

4.127Solução de goma xantana deslocando óleo - Eficiência em função da velocidade adimensional.

4.128Solução de goma xantana deslocando óleo - Eficiência em função da viscosidade adimensional.

4.129Solução de goma xantana deslocando óleo - Viscosidade adimensional em função da taxa de cisalhamento adimensional.

4.130Solução de goma xantana deslocando óleo - Padrões de escoamento, fingers e plug.

C.1 Classificação reológica dos fluidos.

C.2 Curva de viscosidade de um líquido Newtoniano.

C.3 Classificação dos fluidos. 


\section{Lista de tabelas}

4.1 Tabela de resumo da caracterização reológica da poliacrilamida e da goma xantana.

4.2 Parâmetros geométricos da célula de Hele-Shaw. 74

4.3 Propriedade do óleo mineral a $25^{\circ} \mathrm{C}$. 75

4.4 Propriedades das soluções aquosas de glicerina a $25^{\circ} \mathrm{C} \quad 75$

4.5 Parâmetros da poliacrilamida $0,25 \%$ a $25^{\circ} \mathrm{C} . \quad 79$

4.6 Parâmetros da solução de goma xantana $0,40 \%$ a $25^{\circ} \mathrm{C}$. 99

5.1 Taxa de cisalhamento adimensional de transição. 122 
A vida é uma peça de teatro que não permite ensaios. Por isso, cante, chore, dance, ria e viva intensamente, antes que a cortina se feche e a peça termine sem aplausos.

Charles Chaplin 